\title{
AN ASYMPTOTIC FIXED POINT THEOREM FOR A LOCALLY CONVEX SPACE
}

\author{
T. A. BURTON AND D. P. DWIGGINS
}

(Communicated by Kenneth R. Meyer)

\begin{abstract}
The asymptotic fixed point theorem of Horn is extended to a locally convex topological vector space. Applications to differential equations with infinite delay are discussed.
\end{abstract}

1. Introduction. In a series of papers (cf., $[\mathbf{1}, \mathbf{4}, \mathbf{1 1}]$ ) investigators have searched for a natural space in which to discuss the existence of periodic solutions for functional differential equations with infinite delay. From the very start of such investigations it seemed that the proper setting was the metric space $(Y, \rho)$ consisting of the continuous functions $\varphi:(-\infty, 0] \rightarrow R^{n}$ with

$$
\rho(\varphi, \psi)=\sum_{k=1}^{\infty} \frac{2^{-k} \rho_{k}(\varphi, \psi)}{1+\rho_{k}(\varphi, \psi)},
$$

where $\rho_{k}(\varphi, \psi)=\max _{-k \leq s \leq 0}|\varphi(s)-\psi(s)|$ and $|\cdot|$ is any convenient norm on $R^{n}$. This space has two outstanding features useful in these investigations:

(I) If $g:(-\infty, 0] \rightarrow[1, \infty)$ and $\alpha:[0, \infty) \rightarrow[0, \infty)$ are continuous functions, then

$$
S=\{\varphi \in Y|| \varphi(t) \mid \leq g(t) \text { on }(-\infty, 0] \text { and }|\varphi(u)-\varphi(v)| \leq \alpha(|u|+|v|)|u-v|\}
$$

is compact and convex in $(Y, \rho)$.

(II) If the functional differential equation has a proper fading memory, then the Poincaré map is continuous in $(Y, \rho)$.

The Schauder-Tychonov theorem [13] states that if $S$ is a compact convex subset of $(Y, \rho)$ and if $P: S \rightarrow S$ is continuous, then $P$ has a fixed point. Periodic solutions correspond to fixed points of the Poincare map. In particular, if $x(t, \varphi)$ is the solution of a functional differential equation having initial function $\varphi \in Y$, the Poincaré map is defined by

$$
(P \varphi)(t)=x(t+T, \varphi) \text { for }-\infty<t \leq 0 .
$$

Using the properties that solutions are unique and that $x(t+T)$ is a solution when $x(t)$ is a solution, then the task of finding a periodic solution is precisely that of finding a fixed point of $P$ in $Y$.

Detailed discussions of applications for finite delay equations are found in $[\mathbf{7}, \mathbf{8}]$ and for infinite delay in $[\mathbf{1}, \mathbf{4}, \mathbf{5}, \mathbf{6}]$. Continuity of $P$ is extensively discussed in $[\mathbf{4}$

Received by the editors February 23, 1987.

1980 Mathematics Subject Classification (1985 Revision). Primary 34K20.

Key words and phrases. Fixed points, periodic solutions, functional differential equations.

The research of the first author was supported in part by an NSF grant with number NSFDMS-8521408. 
and 10]. Browder [3] gives a substantial list of applications of his asymptotic fixed point theorem. A recent critique is found in [12].

But it frequently happens for ordinary differential equations, for equations with finite delay, for functional differential equations with infinite delay, and for partial differential equations with infinite memory that the Poincaré map does not map $S$ into $S$; instead, some iterate of $P$, say $P^{m}: S \rightarrow S$. While there are several nice asymptotic fixed point theorems for Banach spaces, there does not seem to be a really suitable one for $(Y, \rho)$. Some of Browder's theorems in [3] do hold for $(Y, \rho)$, but they do not appear to fit into the common framework of infinite delay problems.

A fundamental observation for infinite delay problems is that the set $S$ of initial functions must be unbounded; that is, for $\varphi \in S$, then the possibility that $|\varphi(s)| \rightarrow$ $\infty$ as $s \rightarrow-\infty$ must be allowed. And it is this fact that makes property (I) of $(Y, \rho)$ so important. Investigators have been able to embed such $S$ into a Banach space with weighted norm and then apply Horn's asymptotic fixed point theorem. One of the points of this note is that Horn's fixed point theorem may be extended so as to apply to $S$ in $(Y, \rho)$ directly. Moreover, the new result has application to the theory of compact attractors for partial differential equations with infinite memory.

Schauder's theorem and Browder's asymptotic fixed point theorem are proved by a reduction to finite dimensions and application of Browder's fixed point theorem. Here, we embed our sets from $(Y, \rho)$ in an appropriate Banach space and then apply Horn's theorem.

2. The main result. Horn's theorem [9] may be stated as follows.

THEOREM (HORN). Let $S_{0} \subset S_{1} \subset S_{2}$ be convex subsets of a Banach space $X$, with $S_{0}$ and $S_{2}$ compact and $S_{1}$ open relative to $S_{2}$. Let $P: S_{2} \rightarrow X$ be continuous and for some integer $m>0$ let

(a) $P^{j}\left(S_{1}\right) \subset S_{2}, 1 \leq j \leq m-1$, and

(b) $P^{j}\left(S_{1}\right) \subset S_{0}, m \leq j \leq 2 m-1$.

Then $P$ has a fixed point in $S_{0}$.

Notation. (i) $(Y, \rho)$ is the space defined in the Introduction.

(ii) $G$ is the set of continuous scalar functions $g:(-\infty, 0] \rightarrow[1, \infty), g(r) \rightarrow \infty$ as $r \rightarrow-\infty$, and $g$ is decreasing.

(iii) For $g \in G$ then $\left(X_{g},|\cdot|_{g}\right)$ denotes the Banach space of $\varphi \in Y$ for which

$$
|\varphi|_{g}=\sup _{-\infty<s \leq 0}|\varphi(s)| / g(s)
$$

exists.

THEOREM. Horn's theorem is true for $(Y, \rho)$.

PROOF. The proof proceeds by way of four lemmas, followed by a short argument. These lemmas are proved using the relation between convergence in $(Y, \rho)$ and in $\left(X_{g},|\cdot|_{g}\right)$.

LEMMA 1. Let $S \subset Y$ be given and suppose there exists $g \in G$ such that $|\varphi|_{g} \leq 1$ for all $\varphi \in S$. (Then $S \subset X_{g}$.) Let $\varphi \in S$ and a sequence $\left\{\varphi_{n}\right\} \subset S$ be given.

(i) If $\left|\varphi-\varphi_{n}\right|_{g} \rightarrow 0$ as $n \rightarrow \infty$, then $\rho\left(\varphi, \varphi_{n}\right) \rightarrow 0$ as $n \rightarrow \infty$.

(ii) The converse of (i) is false. 
(iii) Let $h=g^{1 / 2}$. (Then $h \in G$ and $X_{h} \subset X_{g}$.) Under the stronger assumption that $|\varphi|_{h} \leq 1$ for all $\varphi \in S$, the converse of (i) is true.

PROOF. (i) Let $\varepsilon>0$ be given and choose a positive integer $M$ with

$$
\sum_{k=M+1}^{\infty} 1 / 2^{k}<\varepsilon / 2 \text {. }
$$

Since $\left|\varphi-\varphi_{n}\right|_{g} \rightarrow 0$, there is an $N>M$ with $\left|\varphi-\varphi_{n}\right|_{g}<\varepsilon / 2 g(-M)$ for $n \geq N$. Thus, for $n \geq N$ and $1 \leq k \leq M$ we have

$$
\begin{aligned}
\varepsilon / 2 g(-M) & >\sup _{-\infty<t \leq 0}\left|\varphi(t)-\varphi_{n}(t)\right| / g(t) \\
& \geq \max _{-k \leq t \leq 0}\left|\varphi(t)-\varphi_{n}(t)\right| / g(t) \\
& \geq \max _{-k \leq t \leq 0}\left|\varphi(t)-\varphi_{n}(t)\right| / g(-M) \\
& =(1 / g(-M)) \rho_{k}\left(\varphi, \varphi_{n}\right),
\end{aligned}
$$

and so $n \geq N$ implies that $\rho_{k}\left(\varphi, \varphi_{n}\right)<\varepsilon / 2$ for $1 \leq k \leq M$. Therefore, $n \geq N$ implies that

$$
\begin{aligned}
\rho\left(\varphi, \varphi_{n}\right) & =\sum_{k=1}^{M} \frac{2^{-k} \rho_{k}\left(\varphi, \varphi_{n}\right)}{1+\rho_{k}\left(\varphi, \varphi_{n}\right)}+\sum_{k=M+1}^{\infty} \frac{2^{-k} \rho_{k}\left(\varphi, \varphi_{n}\right)}{1+\rho_{k}\left(\varphi, \varphi_{n}\right)} \\
& \leq \sum_{k=1}^{M} 2^{-k} \rho_{k}\left(\varphi, \varphi_{n}\right)+\sum_{k=M+1}^{\infty} 2^{-k} \\
& <\sum_{k=1}^{M} 2^{-k} \frac{\varepsilon}{2}+\frac{\varepsilon}{2}<\varepsilon,
\end{aligned}
$$

and so $\rho\left(\varphi, \varphi_{n}\right) \rightarrow 0$ as $n \rightarrow \infty$. This proves (i).

(ii) Given $g \in G$, let $g_{n}(t)=g(t)$ for $-n \leq t \leq 0$ and $g_{n}(t)=g(-n)$ for $-\infty<t<-n$. Then

$$
\rho\left(g, g_{n}\right)=\sum_{k=n+1}^{\infty} \frac{2^{-k} \rho_{k}\left(g, g_{n}\right)}{1+\rho_{k}\left(g, g_{n}\right)} \leq \sum_{k=n+1}^{\infty} 2^{-k}
$$

which tends to zero as $n \rightarrow \infty$. Also, $\left|g_{n}\right|_{g} \leq 1$ for all $n \geq 1$, but $\left|g-g_{n}\right|_{g}=1$ for all $n \geq 1$.

(iii) If $\rho\left(\varphi, \varphi_{n}\right) \rightarrow 0$ as $n \rightarrow \infty$, then $\rho_{k}\left(\varphi, \varphi_{n}\right) \rightarrow 0$ as $n \rightarrow \infty$ for all $k \geq 1$. Also, since $\lim _{r \rightarrow-\infty} h(r)=+\infty$ and $h$ is nonincreasing, there is an integer $M>0$ such that $-\infty<r<-M$ implies that $1 / h(r)<\varepsilon / 2$.

Let $N$ be so large that $n \geq N$ implies that $\rho_{M}\left(\varphi, \varphi_{n}\right)<\varepsilon g(0)$. Then $-M \leq t \leq 0$ implies that

$$
\begin{aligned}
\left|\varphi(t)-\varphi_{n}(t)\right| / g(t) & \leq\left|\varphi(t)-\varphi_{n}(t)\right| / g(0) \\
& \leq \rho_{M}\left(\varphi, \varphi_{n}\right) / g(0)<\varepsilon
\end{aligned}
$$

for $n \geq N$. Also, $-\infty<t<-M$ implies that

$$
\begin{aligned}
\left|\varphi(t)-\varphi_{n}(t)\right| / g(t) & \left.\leq \| \varphi(t)|+| \varphi_{n}(t) \mid\right] / g(t) \\
\leq 2 h(t) / h^{2}(t) & =2 / h(t)<2 \varepsilon / 2=\varepsilon
\end{aligned}
$$


Therefore, $n \geq N$ implies that

$$
\left|\varphi-\varphi_{n}\right|_{g}=\sup _{-\infty<t \leq 0}\left|\varphi(t)-\varphi_{n}(t)\right| / g(t)<\varepsilon
$$

and so $\left|\varphi-\varphi_{n}\right|_{g} \rightarrow 0$ as $n \rightarrow \infty$. This completes the proof of Lemma 1 .

LEMMA 2. If $S$ is compact in $(Y, \rho)$, then there exists a $g \in G$ such that $S \subset X_{g}$ and $S$ is compact in $\left(X_{g},|\cdot|_{g}\right)$.

ProOF. Construct a function $\tilde{g} \in G$ such that $\varphi \in S$ implies that $|\varphi(t)| \leq \tilde{g}(t)$.

This can be done as follows. Define $h:(-\infty, 0] \rightarrow[0, \infty)$ by $h(t)=\sup _{\varphi \in S}|\varphi(t)|$. Since $S$ is compact, $h$ is well defined and continuous on each interval $[-n, 0]$ (by Ascoli's theorem), and so $h$ is well defined and continuous on $(-\infty, 0]$. Define $\hat{h}:(-\infty, 0] \rightarrow[0, \infty)$ by $\hat{h}(t)=\max _{t \leq s \leq 0} h(s)$. Then $\hat{h}$ is continuous, nonnegative, and nonincreasing. Also, $\varphi \in S$ implies that $|\varphi(t)| \leq \hat{h}(t)$. Set $\tilde{g}(t)=$ $\max \left\{\hat{h}(t), e^{-t}\right\}$.

Now, define $g(t)=(\tilde{g}(t)+1)^{2}$ so that $S \subset X_{g}$. Suppose that $\left\{\varphi_{n}\right\} \subset S$. Since $S$ is compact in $(Y, \rho)$, there is a subsequence $\left\{\varphi_{n_{j}}\right\} \subset\left\{\varphi_{n}\right\}$ and $\varphi \in S$ such that $\rho\left(\varphi_{n_{j}}, \varphi\right) \rightarrow 0$ as $n_{j} \rightarrow \infty$. But this implies that $\left|\varphi_{n_{j}}-\varphi\right|_{g} \rightarrow 0$ as $n_{j} \rightarrow \infty$ by Lemma 1. Hence, $S$ is compact in $\left(X_{g},|\cdot|_{g}\right)$. This completes the proof.

LEMMA 3. Given $g \in G$, the topology on $X_{g}$ is finer than the (relative) topology on $(Y, \rho)$.

Proof. Let $\varphi \in X_{g}$ and $\varepsilon>0$ be given. Choose $N$ so that $\sum_{k=N+1}^{\infty} 2^{-k}<\varepsilon / 2$. Consider $U_{g}(\varphi, \delta)=\left\{\psi \in X_{g}:|\varphi-\psi|_{g}<\delta\right\}$, where $\delta=\varepsilon / 2 g(-N)$. If $\psi \in U_{g}(\varphi, \delta)$ and $1 \leq k \leq N$, then

$$
\max _{-k \leq t \leq 0}|\varphi(t)-\psi(t)| / g(-N) \leq \max _{-k \leq t \leq 0}|\varphi(t)-\psi(t)| / g(t) \leq|\varphi-\psi|_{g}<\delta
$$

so that

$$
\rho_{k}(\varphi, \psi)<\delta g(-N)=\varepsilon / 2
$$

This yields $\rho(\varphi, \psi)<\varepsilon$. Hence, $U_{g}(\varphi, \delta) \subset U_{\rho}(\varphi, \varepsilon)$, where $U_{\rho}$ is defined analogously to $U_{g}$; by the Hausdorf criterion $\left[14\right.$, p. 35] every set $U \subset X_{g}$ which is open in the relative topology of $(Y, \rho)$ is also open in $\left(X_{g},|\cdot|_{g}\right)$.

LEMMA 4. Suppose $S \subset Y$ is compact in $(Y, \rho)$ and $P: S \rightarrow Y$ is continuous in $(Y, \rho)$. Then there exists $g \in G$ such that $P: S \rightarrow X_{g}$ is continuous in $\left(X_{g},|\cdot|_{g}\right)$.

Proof. From Lemma 2 there exists $g_{1} \in G$ such that $S \subset X_{g_{1}}$. Also, since $S$ is compact in $(Y, \rho)$ and $P: S \rightarrow Y$ is continuous in $(Y, \rho)$, the image $P(S)$ is compact in $(Y, \rho)$; thus, there exists $g_{2} \in G$ such that $P(S) \subset X_{g_{2}}$. Let $g=g_{1}+g_{2}$. Then $S \subset X_{g}$ and $P(S) \subset X_{g}$, and $P: S \rightarrow X_{g}$. Moreover, $g_{1}$ and $g_{2}$ can be chosen such that $\rho\left(\varphi, \varphi_{n}\right) \rightarrow 0$ if and only if $\left|\varphi-\varphi_{n}\right|_{g} \rightarrow 0$.

Now, let $\varphi \in S$ be given and suppose that $\left\{\varphi_{n}\right\} \subset S$ with $\left|\varphi-\varphi_{n}\right|_{g} \rightarrow 0$. Then $\rho\left(\varphi, \varphi_{n}\right) \rightarrow 0$ which implies that $\rho\left(P \varphi, P \varphi_{n}\right) \rightarrow 0$ since $P$ is continuous in $(Y, \rho)$. Thus, $\left|\varphi-\varphi_{n}\right|_{g} \rightarrow 0$ implies that $\rho\left(P \varphi, P \varphi_{n}\right) \rightarrow 0$ so that $\left|P \varphi-P \varphi_{n}\right|_{g} \rightarrow 0$. This means that $P$ is continuous in $\left(X_{g},|\cdot|_{g}\right)$. The proof is complete.

We can now finish the proof of the theorem. From Lemma 2 there exists $g_{1} \in G$ such that $S_{0}$ and $S_{2}$ are compact in $\left(X_{g_{1}},|\cdot|_{g_{1}}\right)$. By Lemma 4 there exists $g_{2} \in G$ such that $P: S_{2} \rightarrow X_{g_{2}}$ is continuous in $\left(X_{g_{2}},|\cdot| g_{2}\right)$. Let $g=g_{1}+g_{2}$. Then $S_{0}$ 
and $S_{2}$ are compact in $X_{g_{1}} \subset X_{g}$, and $P: S_{2} \rightarrow X_{g}$ is still continuous in $\left(X_{g},|\cdot|_{g}\right)$. Also, from Lemma 3 we see that $S_{1}$ is open relative to $S_{2}$ in $\left(X_{g},|\cdot| g\right)$. Conditions (a) and (b) remain unchanged, so from Horn's theorem, $P$ must have a fixed point. This completes the proof.

\section{BIBLIOGRAPHY}

1. O. Arino, T. A. Burton, and J. Haddock, Periodic solutions of functional differential equations, Proc. Roy. Soc. Edinburgh Sect. A 101A (1985), 253-271.

2. F. E. Browder, On a generalization of the Schauder fixed point theorem, Duke Math. J. 26 (1959), 291-303.

3.

4. T. A. Burton, Stability and periodic solutions of ordinary and functional differential equations, Academic Press, Orlando, 1985.

5. __ Periodic solutions of nonlinear Volterra equations, Funkcial. Ekvac. 27 (1985), 301-317.

6. __ Periodic solutions of functional differential equations, J. London Math. Soc. (2) (6) IV (1985), 31-39.

7. J. K. Hale, Theory of functional differential equations, Springer-Verlag, New York, 1977.

8. J. K. Hale and O. Lopes, Fixed point theorems and dissipative processes, J. Differential Equations 13 (1973), 391-402.

9. W. A. Horn, Some fixed point theorems for compact maps and flows in Banach spaces, Trans. Amer. Math. Soc. 149 (1970), 391-404.

10. T. Kaminogo, Continuous dependence of solutions for integrodifferential equations with infinite delay, preprint.

11. P. Massatt, Stability and fixed points of point-dissipative systems, J. Differential Equations 40 (1981), 217-231.

12. R. Sine, Remarks on a paper of Horn, preprint.

13. D. R. Smart, Fixed point theorems, Cambridge Univ. Press, London and New York, 1980.

14. S. Willard, General topology, Addison-Wesley, Reading, Mass., 1970.

Department of Mathematics, Southern Illinois University, Carbondale, ILLINOIS 62901 